\title{
High-output heart failure resulting from a remote traumatic arteriovenous fistula
}

\author{
Kibar Yared MD FRCPC, Aaron L Baggish MD, Malissa J Wood MD FACC
}

\begin{abstract}
A
55-year-old man who received a gunshot wound to the right buttock 30 years previously was admitted to the hospital with complaints of progressive exertional dyspnea, nausea, vomiting and right flank pain. On admission, his physical examination demonstrated a blood pressure of 106/73 $\mathrm{mmHg}$, an irregular heart rate of 170 beats/min and an oxygen saturation of $99 \%$ on room air. Auscultation revealed bibasilar pulmonary rales and a bruit in the right lower quadrant of the abdomen where palpation demonstrated a tender, pulsatile abdominal mass. Atrial fibrillation with a rapid ventricular response was evident on an electrocardiogram, and an echocardiogram demonstrated new marked biventricular dilation with signs of right ventricular volume overload and a left ventricular ejection fraction of $50 \%$. Subsequent contrast-enhanced computed tomography (CT) scanning revealed cardiomegaly, bilateral pleural effusions, a dilated inferior vena cava and hepatic veins as well as an arteriovenous fistula arising from the right internal iliac artery (RIIA) and communicating with the right common iliac vein (RCIV). There was also evidence of a $12.4 \mathrm{~cm} \times 10.1 \mathrm{~cm} \times 12.3 \mathrm{~cm}$ aneurysmal dilation of the RCIV (Figures 1 and 2). Numerous gunshot pellets were noted throughout the abdomen.

The patient underwent catheterization, at which point, his cardiac output was measured, by the thermodilution method, to be 13.3 L/min. Several percutaneous attempts at exclusion of the RIIA using an Amplatzer vascular plug device (AGA Medical Corporation, USA) as well as several Nester coils (Cook Medical Inc, USA) were unsuccessful. Eventually, the fistula was occluded using a $16 \mathrm{~mm}$ Amplatzer atrial septal defect occluder (AGA Medical, USA). Nevertheless, persistent flow from the RIIA into the right internal iliac vein was demonstrated; however, this was expected to diminish as anticoagulation was withdrawn. The patient's hospital course was further complicated by thrombosis of the venous aneurysm with resultant thrombocytopenia, hematuria and obstructive jaundice. A follow-up angiogram revealed persistent opacification of the fistula from the RIIA. Together with a vascular surgical team, the patient subsequently underwent placement of an endovascular graft converter (Zenith Flex, Cook Medical Inc) in the RIIA (Figure 3) and a permanent inferior vena cava filter (Trapease; Johnson \& Johnson, USA) in the RCIV (Figure 4). Although minimal residual flow into the RCIV remained postintervention, it had been reduced significantly by the described interventions.
\end{abstract}

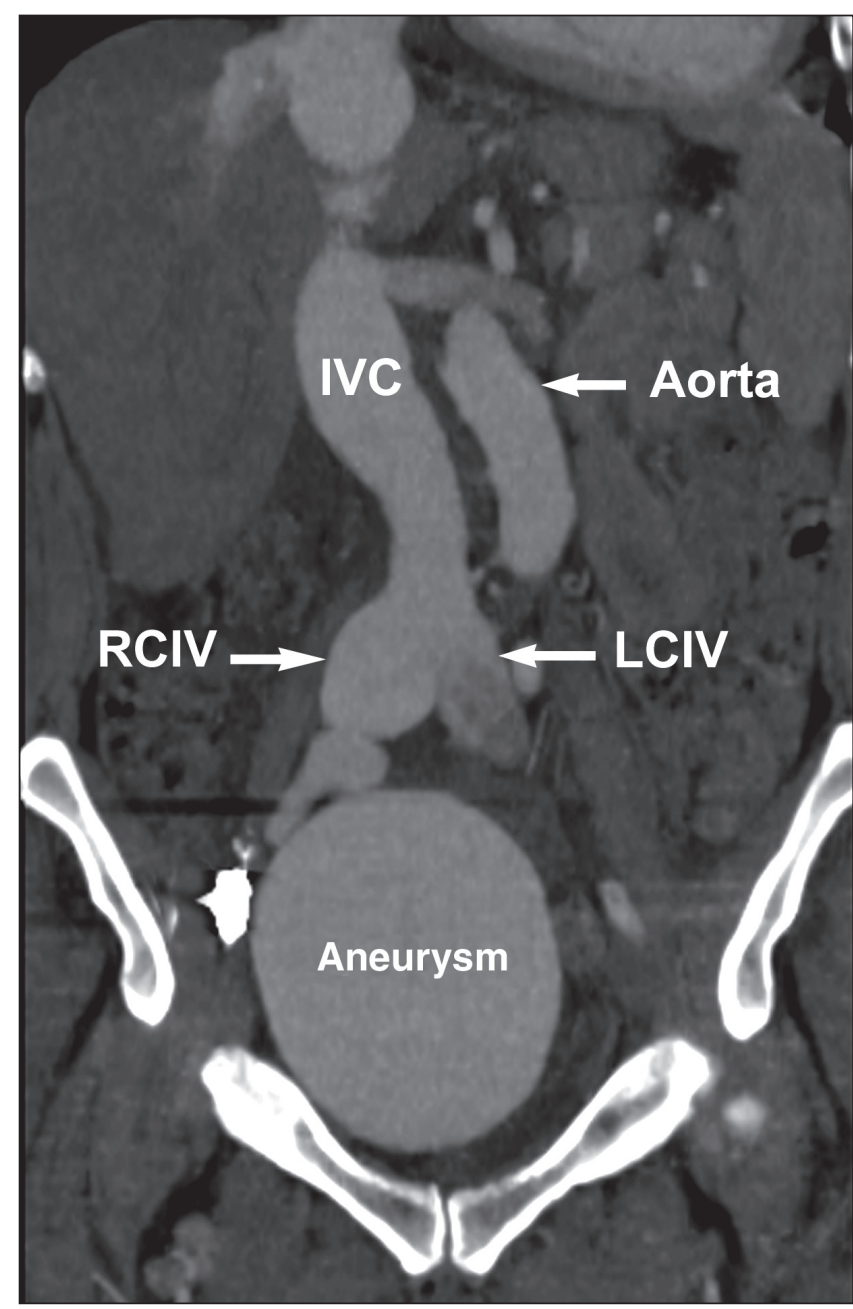

Figure 1) Abdominopelvic computed tomography scan demonstrating a $12.4 \mathrm{~cm} \times 10.1 \mathrm{~cm} \times 12.3 \mathrm{~cm}$ aneurysmal dilation of the right common iliac vein (RCIV). IVC Inferior vena cava; LCIV Left common iliac vein

Department of Medicine, Division of Cardiology, Massachusetts General Hospital, Harvard Medical School, Boston, Massachusetts, USA

Correspondence: Dr Kibar Yared, Department of Medicine, Division of Cardiology, Massachusetts General Hospital, Yawkey 5E, 55 Fruit Street, Boston,

Massachusetts 02114-2696, USA. Telephone 617-724-7738, fax 617-643-1639, e-mail kyared@partners.org

Received for publication May 13, 2008. Accepted June 21, 2008 


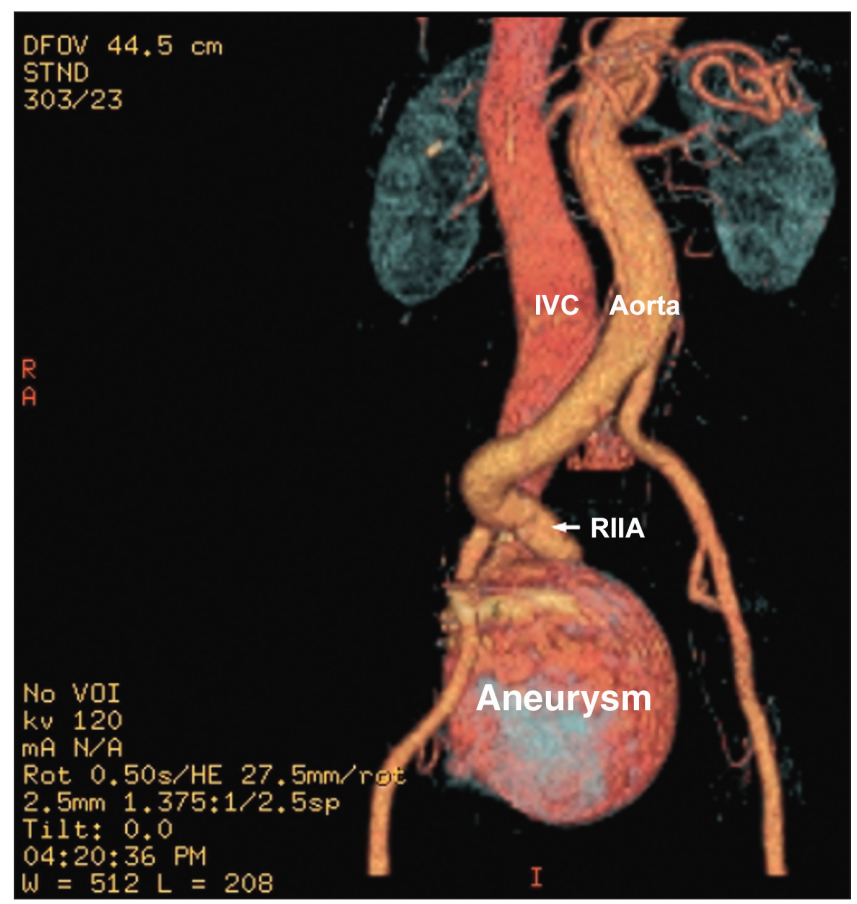

Figure 2) Abdominopelvic computed tomography scan with three-dimensional reconstruction. IVC Inferior vena cava; RIIA Right internal iliac artery

Three weeks after endovascular graft placement, collateral flow from the right L4 lumbar artery into the RIIA was demonstrated by CT angiography with continued opacification of the RCIV from the RIIA. Percutaneous coiling (Diamond Vortex coils; Boston Scientific, USA) of the L4 lumbar artery was then attempted, which resulted in successful exclusion of the RIIA. No further flow into the RIIA could be demonstrated on fluoroscopy. Two weeks later, the patient was pain free and ambulating without complaints of dyspnea. At that time, an echocardiogram demonstrated reduced left and right ventricular dimensions as well as normal biventricular systolic function. A follow-up CT of the patient's abdomen and pelvis showed a reduction in the size of the aneurysm $(9.3 \mathrm{~cm} \times 9.0 \mathrm{~cm})$ as well as absent flow within the fistula and into the RIIA from the L4 lumbar artery.

The present case highlights two important points. Previously healthy, young men presenting with high-output congestive heart failure and a history of penetrating injuries to the abdomen and/or pelvis should undergo thorough testing for arteriovenous fistulas. In addition, chronic arteriovenous fistulas often involve complex arteriovenous anatomy, as did our patient, often complicating percutaneous closure. For this reason, meticulous preprocedural evaluation is of the utmost importance. As well, close collaboration between vascular surgery and interventional cardiology/radiology is necessary to facilitate the selection of the most appropriate intervention on a case-by-case basis.

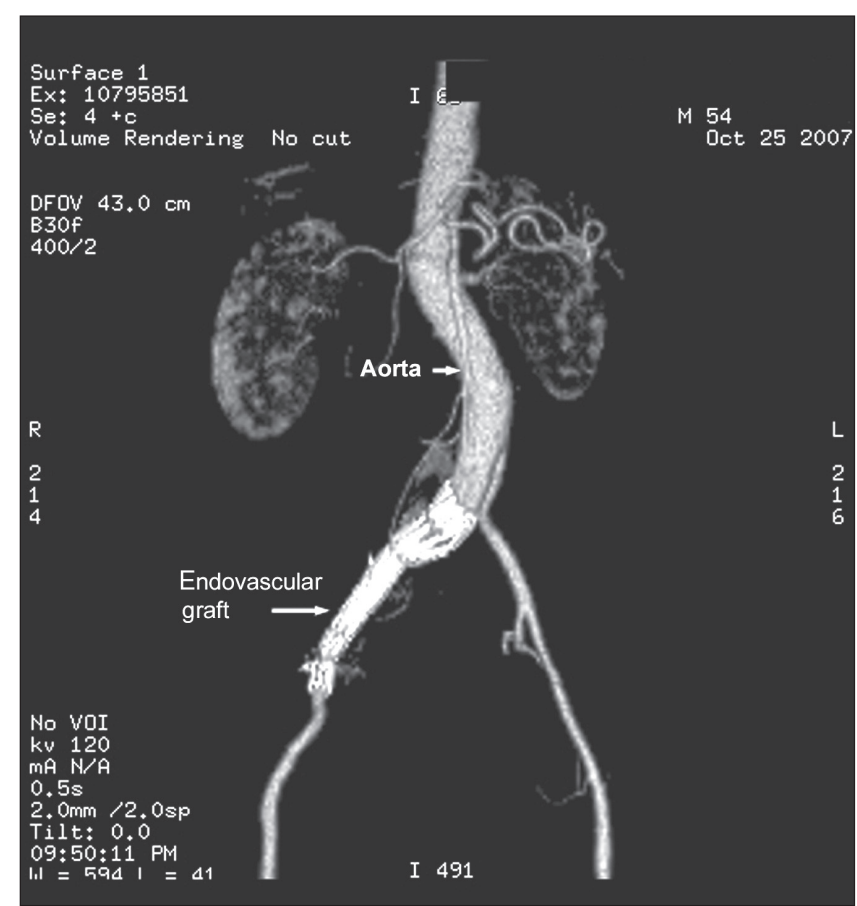

Figure 3) Abdominopelvic computed tomography scan with three-dimensional reconstruction postendovascular graft implantation in the right internal iliac artery

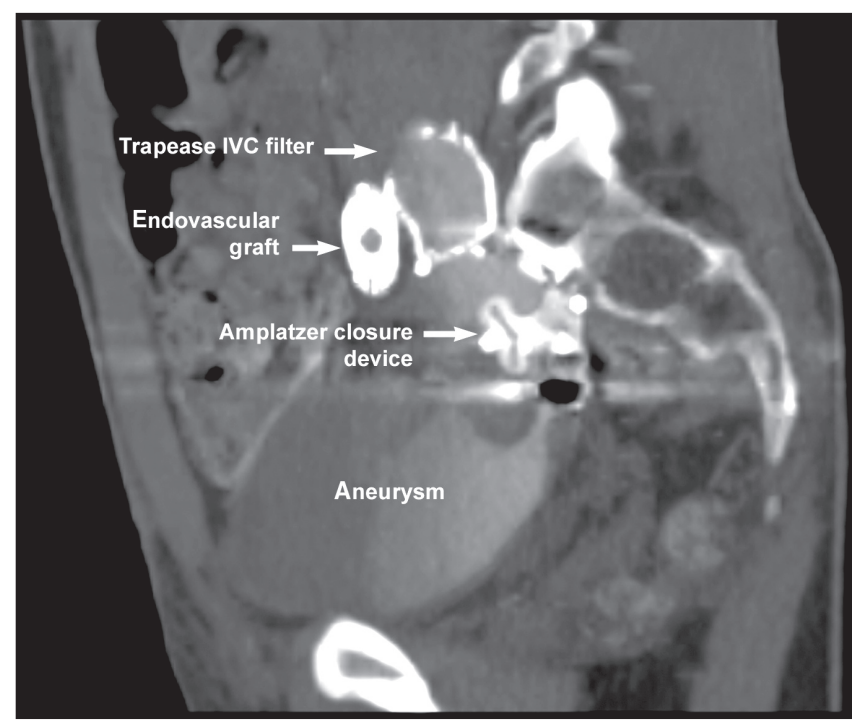

Figure 4) Sagittal cut of an abdominopelvic computed tomography scan demonstrating a Trapease filter (Johnson $\mathbb{E}$ Johnson, USA) in the inferior vena cava (IVC), the endovascular graft in cross-section and an Amplatzer closure device (AGA Medical Corporation, USA). Numerous gunshot pellets are evident throughout the abdomen 